\title{
Effects of differences in timbre on sequential grouping
}

\author{
RHODRI CUSACK and BRIAN ROBERTS \\ University of Birmingham, Birmingham, England
}

\begin{abstract}
Differences in the timbre of sounds in a sequence can affect their perceptual organization. Using a performance measure, Hartmann and Johnson (1991) concluded that streaming could be predicted primarily by the extent to which sounds were passed by different peripheral channels. However, results from a rating task by Dannenbring and Bregman (1976) suggested that sounds in the same spectral region (passed by the same peripheral channels) can be allocated to different streams. In Experiment 1, it was found, using an interleaved melody task, that target sounds could be selected from distractors in the same spectral region more easily when they differed in timbre. This finding might result from primitive stream segregation or schema-driven selection, but not from peripheral channeling. In Experiment 2, a rhythm discrimination task was used, requiring the sounds to be integrated for good performance. Differences in timbre impaired performance, indicating the occurrence of primitive stream segregation.
\end{abstract}

The sound arriving at the ear is often a mixture originating from several sources. A key process in the interpretation of such a composite sound is to partition it into streams, each corresponding to a different source. There are two aspects to the solution of this scene analysis problem. One is the separation of concurrent sounds, and the other is the linking together of successive sounds from a common source (Bregman, 1990). The present study relates to the latter problem of sequential grouping. The effect of differences in timbre on auditory streaming is used to evaluate Hartmann and Johnson's (1991) peripheralchanneling hypothesis, and the contributions of bottomup and top-down grouping processes are explored.

Hartmann and Johnson (1991) proposed that most sequential streaming effects can be explained by differences in the peripheral channeling of sounds. It is well established that sounds are analyzed by frequency in the cochlea and that auditory nerve fibers are tuned by frequency. Hartmann and Johnson used the name peripheral channeling for this selection by frequency and by ear. They proposed that sounds passed by peripheral channels that are more similar (i.e., in a more similar spectral region and presented to the same ear) are less likely to segregate perceptually from one another. This proposal is appealing, because it is a simple extension of mechanisms that are well established neurophysiologically. Indeed, there have been recent attempts to develop computer models on the

We thank Peter Bailey, Jeff Brunstrom, and Mike Harris for their helpful comments and criticism. Our thanks also go to Al Bregman and two anonymous reviewers for their comments on an earlier version of this manuscript. Correspondence concerning this article should be addressed to R. Cusack, MRC-Cognition and Brain Sciences Unit, 15 Chaucer Road, Cambridge CB2 2EF, England (e-mail: rhodri.cusack@mrc-cbu. cam.ac.uk) basis of this idea (e.g., Beauvois \& Meddis, 1991; McCabe \& Denham, 1997).

Hartmann and Johnson's (1991) model was inspired by a set of experiments using an interleaved melody task similar to that used by Dowling (1973, Experiment 1). Two melodies (A and $B$ ) were said to be interleaved if the notes were played in the order A1, B1, A2, B2, and so on. On each trial, listeners were asked to identify two familiar melodies that were interleaved. A comparison condition was included in which both the melodies consisted of pure tones. Contrasts between the acoustic attributes of the melodies were introduced, and the effects on performance were measured. A variety of conditions were tested, in which the sounds differed in their temporal envelopes, spectral composition, interaural time delay, or ear of presentation. They concluded that "What does seem clear is that peripheral channeling is the dominant characteristic seen in stream segregation" (p. 177). In Condition 12, for example, one of the melodies consisted of pure tones, and the other of two-tone complexes. These sounds had a distinctly audible difference in timbre, but no enhanced stream segregation was found as a result. This is as predicted by the peripheral-channeling hypothesis.

Hartmann and Johnson's (1991) hypothesis is consistent with the results of many previous studies of sequential grouping. It is well established that sequences of pure tones are more likely to segregate if they are spread over a wider range of frequencies and, hence, peripheral channels (e.g., Bregman \& Campbell, 1971; Van Noorden, 1975). The peripheral-channeling model also predicts well the results of studies of the effect of differences in other acoustic attributes on streaming. For example, Van Noorden played a sequence comprising two complexes with the same, missing, fundamental $(F 0)$ frequency, but with different selections of harmonics (3-5 and 8-10). Van Noor- 


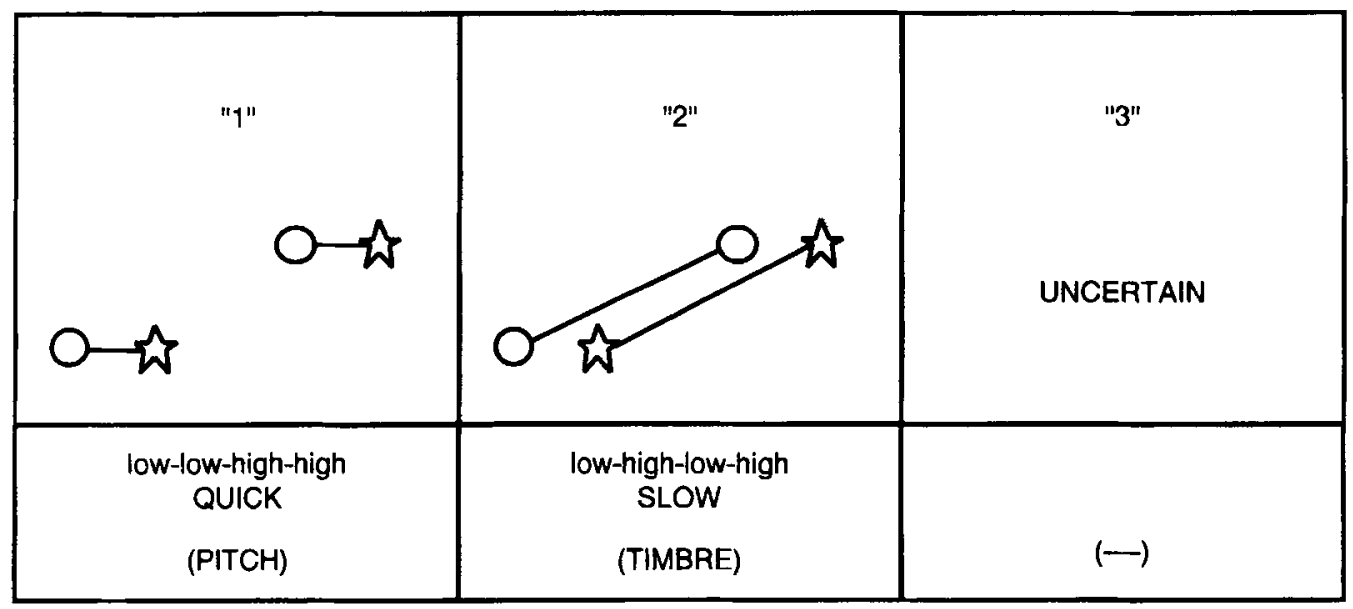

Figure 1. Response labels used by Singh (1987) to denote different perceptual groupings. From "Perceptual Organization of Complex-Tone Sequences: A Tradeoff Between Pitch and Timbre,” by P. G. Singh, 1987, Journal of the Acoustical Society of America, 82, pp. 886-899. Copyright 1987 by the Acoustical Society of America. Adapted with permission.

den found that although the complexes had the same $F 0$ frequency, they still tended to stream. He concluded that differences in spectral region could lead to streaming. In an extension of this study, he found no evidence of a role in streaming for differences in $F 0$ frequency. However, Singh's (1987) investigation of the grouping of sequences of complex tones has demonstrated a role for $F 0$ frequency, as well as for spectral region. Each sound used was defined by a combination of one of seven timbres and one of seven pitches. The timbres were denoted by $T_{1}, T_{2}, \ldots$ $T_{7}$, in which timbre $T_{m}$ consisted of the four harmonics $m$, $m+1, m+2$, and $m+3$. The pitches, chosen to fall on a musical scale, were denoted by $P_{1}, P_{2}, \ldots P_{7}$ and corresponded to fundamental frequencies of $262,330,392$, $524,660,784$, and $1048 \mathrm{~Hz}$, respectively. A repeating sequence of four tones $-T_{2} P_{1}-T_{m} P_{1}-T_{2} P_{n}-T_{m} P_{n}-\ldots$, where $m$ and $n$ were varied between conditions-was played.

This sequence can group perceptually by either pitch or timbre. Listeners were asked to describe the grouping for each trial, using the response labels shown in Figure 1 . The results showed a tradeoff between pitch and timbre, indicating competition between them in determining the perceptual organization of the sequence. When there was a large difference in timbre and a small difference in pitch, the percept was that of grouping by timbre, but when there was a large difference in pitch and a small difference in timbre, the percept was that of grouping by pitch. When there were differences in pitch and timbre of roughly equivalent magnitude (as measured by the difference between their suffix labels), the percept heard was ambiguous. Singh's (1987) results could be explained by two factors: (1) Sounds with a more similar $F 0$ frequency are less likely to segregate, and (2) sounds in a more similar spectral region are less likely to segregate. Hartmann and Johnson (1991, p. 176) also found that listeners can segregate sequences of complex tones with substantial spectral overlap and admitted that streaming cannot actually take place in the peripheral auditory system. However, they suggested that perhaps even this effect is a result of the peripheral channeling of resolved harmonics, which then "carry along" the unresolved components.

Peripheral channeling appears also to offer a potential explanation for many of the results of experiments using more complex stimuli. In studies using sequences of musical instrument sounds, the best predictor of their segregation was found to be the similarity of their spectral center of gravity (Iverson, 1995; Wessel, 1979). It is likely that there will be a strong correlation between the similarity of sounds in their spectral center of gravity and the amount of overlap in their peripheral channeling, and so this finding is consistent with Hartmann and Johnson's (1991) hypothesis. Bregman, Liao, and Levitan (1990) used vowel-like sounds, in a task similar to that of Singh (1987), to investigate the relative roles in streaming of $F 0$ frequency and spectral structure. Their stimuli consisted of complex tones with a single formant peak. It was found that sounds with either a more similar $F 0$ frequency or a more similar formant frequency were more likely to group together. They also found that the sharpness of the formant peaks affected grouping: A difference in center frequency between sharper peaks was found to be more effective in inducing streaming than a difference between broader peaks. Again, in these stimuli, there was a correlation between the difference in formant frequency and sharpness and the amount of overlap in the peripheral channeling of their sounds. Sounds with a larger difference in formant frequency or sharper peaks had less spectral overlap, and so, according to the peripheral-channeling hypothesis, they might be expected to stream more. This is what was observed.

Recently, Singh and Bregman (1997) published results that suggest an important role for an acoustic attribute that 
does not affect the frequency spectrum. They presented sequences of the form $\mathrm{ABA}-\mathrm{ABA}-$, similar to those used by Van Noorden (1975). When heard as a single stream, these sequences are heard as having a characteristic "gallopping" rhythm, which is lost when sounds A and B segregate. Over the course of each trial, Singh and Bregman varied the difference between the $F 0$ frequencies of sounds $\mathrm{A}$ and $\mathrm{B}\left(\Delta F_{0}\right)$ and asked listeners to signal when the sequence "crossed over" from one stream to two streams or vice versa. They varied the number of harmonics (two or four) and the rise and fall times of the sounds (rise/fall $5 / 95 \mathrm{msec}$ or $95 / 5 \mathrm{msec}$ ). Stream segregation ratings were taken in four conditions in which sounds A and B (1) had the same temporal envelope and the same number of harmonics ( $\mathrm{SeSn}),(2)$ were different only in temporal envelope (DeSn), (3) were different only in the number of harmonics (SeDn), and (4) had a different temporal envelope and number of harmonics (DeDn). They found that stream segregation occurred for a smaller $\Delta F_{0}$ in the DeSn condition than in the SeSn condition, even though a difference in temporal envelope has little effect on frequency channeling. Singh and Bregman's results do not demonstrate that streaming can occur in the absence of peripheral channeling, because the $\Delta F_{0}$ used always resulted in some peripheral channels excited only by either the A or the B sounds. However, their results do demonstrate that other factors (specifically, the temporal envelope) can alter the extent of stream segregation. It should be noted that Singh and Bregman did not find greater streaming in the DeDn condition than in the SeDn condition. This was attributed to a floor effect.

Experiments demonstrating stream segregation in the absence of peripheral channeling have been reported by Dannenbring and Bregman (1976). They played a sequence consisting of a pair of narrowband sounds, alternating repeatedly. Listeners were asked to rate both the degree of stream segregation and the degree of perceived temporal overlap of the sounds. Segregated sounds are often heard as overlapping, since their exact temporal relation is difficult to determine (Warren \& Warren, 1970). Given that the sounds used did not physically overlap in any of the conditions in Dannenbring and Bregman's experiments, the perceived overlap was, in most cases, another measure of stream segregation. They investigated (Experiments 2-6) the streaming of (1) two narrowband noises, (2) one narrowband noise and one pure tone, and (3) two pure tones. The noises had a 3-dB bandwidth of 1.5 semitones, equivalent to $90 \mathrm{~Hz}$ for a center frequency of $1000 \mathrm{~Hz}$. The frequency separation of the two sounds was also varied. As was expected (Bregman \& Campbell, 1971; Van Noorden, 1975), it was found that sounds with larger frequency separations showed greater stream segregation. Furthermore, sounds of different timbre (noise/tone) segregated more than those of the same timbre (noise/noise and tone/tone), and the effect was large. Indeed, sounds of different timbre with the same center frequency $(1000 \mathrm{~Hz})$ segregated more than sounds of the same timbre with moderate frequency differences (e.g., center frequencies of 1000 and $1200 \mathrm{~Hz}$ ) and almost as much as those with large frequency differences (e.g., center frequencies of 1000 and $3000 \mathrm{~Hz}$ ). In most cases, the perceived overlap increased as the stream segregation increased. However, the continuity illusion affected the results in some cases, since the pure tones were heard to continue through the noise bands. This was confirmed by a direct rating of the perceived continuity.

Dannenbring and Bregman's (1976) findings contrast with those of Hartmann and Johnson (1991). Glasberg and Moore's (1990) formula estimates an equivalent rectangular bandwidth of $130 \mathrm{~Hz}$ for an auditory filter centered on $1000 \mathrm{~Hz}$. Given that the bandwidth of the noises used was only $90 \mathrm{~Hz}$ for this center frequency, they would have fallen mainly within the same peripheral channel as that for pure tones of the same center frequency. Despite this, pure tones and narrowband noises centered on $1000 \mathrm{~Hz}$ were shown to stream almost as much as pure tones separated by more than an octave, which fall into very different peripheral channels. The origin of the difference in findings is unclear. Perhaps the subjective rating procedure used by Dannenbring and Bregman did not provide a pure measure of streaming. Given the inconclusive evidence concerning the role of timbre differences and peripheral channeling in sequential grouping, the issue was explored further, using a performance measure of the streaming of narrowband sounds within the same frequency range.

\section{EXPERIMENT 1}

\section{Method}

Overview and conditions. A modification of Dowling's (1973) interleaved melody task, with a three-interval two-alternative forced-choice trial structure, was used. The discrimination threshold for a small change (melodic alteration) to all of the tones in a short "melody" was measured when it was interleaved with distractors. The notes of the target melody were pure tones in all the conditions. Relative to the target tones, the distractors were (1) of the same timbre and in the same frequency range (Condition 1, no difference), (2) of a different timbre and in the same frequency range (Condition 2, timbral contrast), or (3) of the same timbre, but in a frequency range one octave higher (Condition 3, frequency contrast). The distractors were pure tones in Conditions 1 and 3 but were narrowband noises in Condition 2 . To calculate an estimate of the spread of excitation evoked by the noises and pure tones, Glasberg and Moore's (1990) formulae were used. The bandwidths of the 3-dB-down points in the excitation patterns for the pure tones and the narrowband noises were 2.40 and 2.95 semitones, respectively. Relative to the range of the melodies (eight semitones), this difference in the spread of excitation was small. Hence, there was only a slightly greater overlap in the frequency channeling of the targets and distractors in Condition 2 than in Condition 1. If sequential sounds can only be segregated when they are passed by different peripheral channels, there should be very little difference in the amount of streaming in Conditions 1 and 2. Performance on the task might be expected to be similar, or perhaps slightly worse, in Condition 2 . In contrast, the targets and distractors were separated by more than an octave in Condition 3 and, hence, were passed by very different peripheral channels. Therefore, strong stream segregation might be expected, leading to better performance in Condition 3 . 


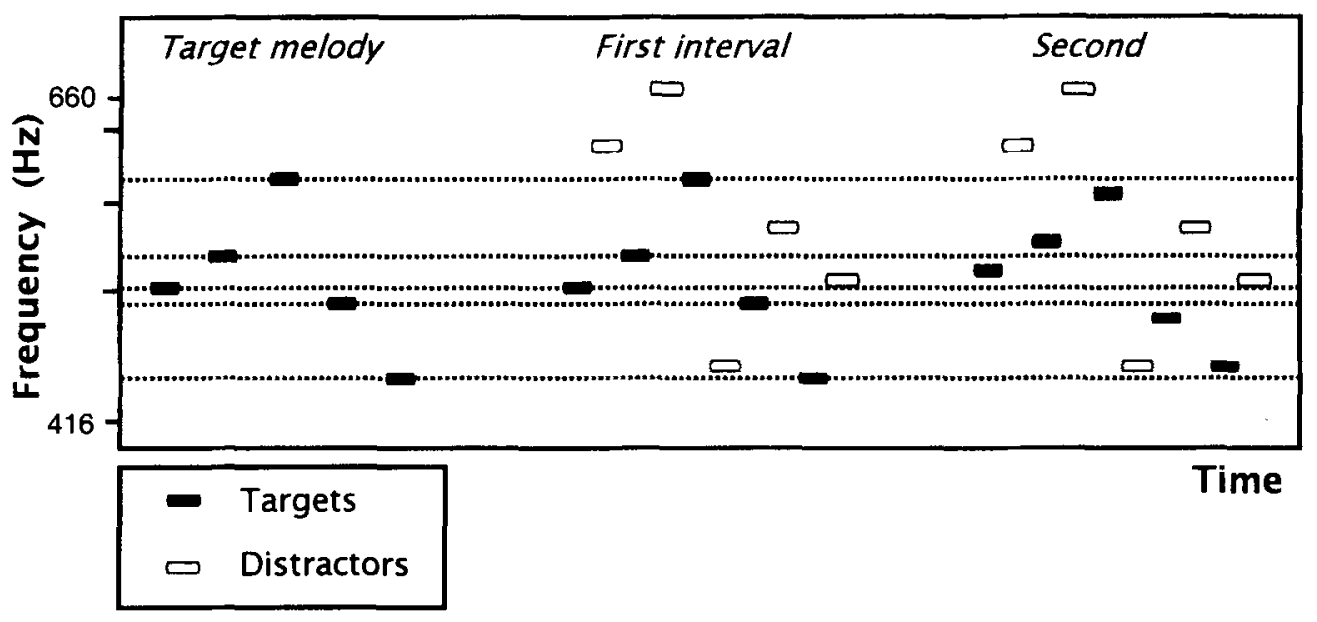

Figure 2. Trial structure of the interleaved melody task.

Subjects. Thirteen students participated in this experiment of about $45 \mathrm{~min}$. The data from 1 student were rejected (see below), giving a total of 12 subjects. All the subjects reported normal hearing.

Apparatus. The stimuli were generated digitally and played at a conversion rate of $16 \mathrm{kHz}$ with a precision of 16 bits via a D/A converter (Data Translation 2823). The stimuli were low-pass filtered (corner frequency $=5.2 \mathrm{kHz}$, roll-off $=100 \mathrm{~dB} / \mathrm{oct}$ ) and presented binaurally over Sennheiser HD-480-II headphones. The levels of the stimuli were set using a programmable attenuator and were calibrated with a sound-level meter (Brüel \& Kjaer Type 2209, equal weighting) connected to the headphones by an artificial ear (Type 4153). The listeners sat in a sound-attenuating chamber (Industrial Acoustics) and responded with a computer keyboard.

Stimuli. Each melody consisted of five pure-tone notes. The frequencies of these notes were calculated in semitone units. There were three stages in the process of melody generation. First, a prototype sequence was generated by randomly selecting the frequency of each note in turn from a rectangular distribution in the range 8-16 semitones above middle-C $(416-660 \mathrm{~Hz})$. This sequence of frequencies can be represented as $F 1, F 2, \ldots F 5$. Second, two melodies were derived from the prototype, one of which was designated the true-target melody and the other of which was designated the false-target melody. This was done so that (1) there was a fixed difference in frequency ( $A$, the melodic alteration size) between corresponding notes in the pair of melodies and (2) the arithmetic mean of each pair of corresponding notes equalled the value for that note position in the prototype sequence. Hence, $F 1$ became the pair of values $F 1-A / 2$ and $F 1+A / 2$, and so forth. Third, the allocation of the higher frequency note to either the trueor the false-target melody was determined randomly for each note position in turn. The stimuli were generated in this way so that the long-term average distribution of notes across frequency was identical for the true- and the false-target melodies.

The distractors were also chosen randomly from the frequency range $8-16$ semitones above middle- $C$ but were constrained so that they were never closer than 2 semitones to the arithmetic mean of the frequencies of the true and false targets preceding or succeeding them. This constraint was introduced because pilot work had shown that two consecutive sounds with very similar center frequencies tended to "pop out" from the sequence. The distractors were pure tones in Condition 1 and noises of 2 semitones in bandwidth in Condition 2 ( $62 \mathrm{~Hz}$ for a center frequency of $524 \mathrm{~Hz}$ ). The distractors were also pure tones in Condition 3 but were transposed upward by one octave.

The narrowband noises were generated digitally by adding equalamplitude sinusoids, with random starting phases, distributed at 2$\mathrm{Hz}$ intervals across the required bandwidth. Both the targets and the distractors were $125 \mathrm{msec}$ in duration, with 10 -msec linear-amplitude onset and offset ramps, and were played without gaps (i.e., at a rate of eight notes per second when interleaved ${ }^{1}$ ). The steady-state portions of the sounds had a level of $77 \mathrm{~dB}$ SPL.

Procedure. Each trial consisted of three intervals. The trial structure is shown in Figure 2. First, the target melody was played without distractors. Then, there were two intervals in which a melody was interleaved with distractors. In one, the melody was the true target; in the other, the target underwent melodic alteration (i.e., each tone was randomly shifted up or down by a specified amount) to give a false target. The distractors in these two intervals had the same frequencies and order. The listeners were asked to identify the interleaved presentation in which the true target was present. The size of the melodic alteration was varied, using Levitt's (1971) two-up one-down adaptive procedure to converge on the $71 \%$ correct melodic-alteration threshold. A practice block of four trials from each of the three conditions, presented in random order, was given to each subject. There then followed three adaptive staircase runs, one for each condition, which were counterbalanced in order across subjects. The parameters for the adaptive procedure were estimated through simulation and tested in pilot work. At the start of a staircase, the melodic-alteration size was set to $1.6 \mathrm{semi-}$ tones. Throughout an adaptive run, it was constrained between 0.04 and 1.8 semitones. The initial step size was 0.16 semitones. This was halved after 3 and again after 6 turning points. The staircase was terminated on the 12th turning point, and the average of the final 6 turning points was taken as the melodic-alteration threshold. The data from a subject were rejected if the average melodic-alteration threshold in two or more of the conditions was at floor (defined as being greater than 1.6 semitones). The data from 1 subject were rejected on this basis and were replaced with data from a new subject presented with the conditions in the same order.

\section{Results}

Figure 3 shows the melodic-alteration discrimination thresholds. The data were analyzed, using a one-way 


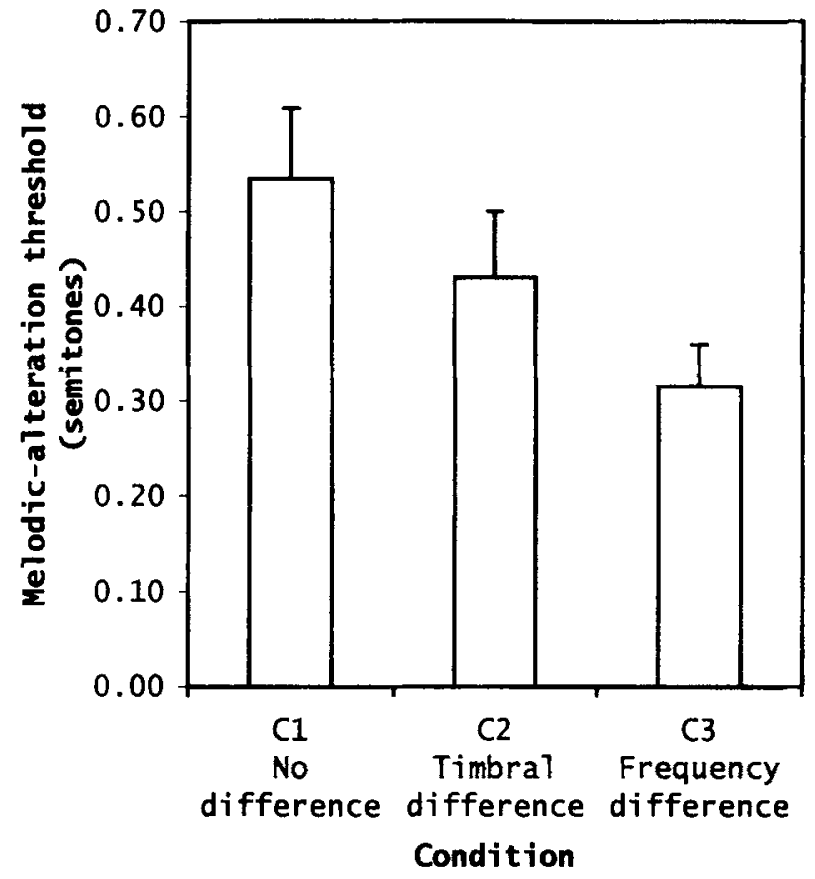

Figure 3. Melodic-alteration thresholds in the three conditions of Experiment 1. A lower threshold corresponds to better performance. The mean performance over 12 subjects, with one standard error, is shown.

within-subjects analysis of variance (ANOVA), and the effect of condition was found to be highly significant $[F(2,22)=18.81, p<.001]$. Fisher's test was used to perform all possible a posteriori comparisons. Thresholds were reduced by $40 \%$ when there was a frequency contrast between the distractors and the target notes [Condition 3 vs. Condition 1: $\left.t^{\prime}(11)=5.74, p<.001\right]$. A smaller, but still significant, reduction of $20 \%$ was found when there was a timbral contrast [Condition 2 vs. Condition 1 : $\left.t^{\prime}(11)=2.61, p<.02\right]$. The difference in thresholds between Conditions 2 and 3 was also significant $\left[t^{\prime}(11)=\right.$ $3.13, p<.005]$.

\section{Discussion}

Hartmann and Johnson (1991) found that pure tones and narrowband sounds (two-tone complexes) in the same frequency region did not segregate from one another. They concluded that sounds are likely to show little stream segregation in the absence of peripheral channeling. This is not consistent with the results presented here, and it is not clear how the difference in narrowband sounds used (noisebands instead of two-tone complexes) might account for this discrepancy. Although it is true that a one-octave frequency difference did lead to the largest increase in performance on the task, the improvement seen when there was a timbral contrast was highly reliable and was evident for all but 2 of the 12 subjects. The average size of the improvement in melodic-alteration threshold for a timbral contrast was fully half the size of that seen with a frequency contrast. Therefore, it seems likely that the observed effect of timbral contrast is not of a different order to that seen with a frequency contrast.

\section{INTERIM DISCUSSION Primitive Stream Segregation or Schema-Based Selection?}

Consideration should be given as to what is required for good performance on the interleaved melody task. In particular, the question of whether or not bottom-up primitive stream segregation is necessary to perform the task should be addressed. If it is not, then what is really being measured? Bregman (1990, Chap. 4) discusses the role of attention in the perceptual organization of sounds. He accepts that we can select familiar sounds when there are no simple acoustic cues, using knowledge about their form. These top-down processes that use knowledge about patterns in the environment to perform such pattern recognition are often referred to as schemas. Part of the definition of primitive strean segregation processes is that they are automatic. Perhaps the most powerful demonstrations of the existence of primitive stream segregation are those in which it has been shown that sounds are segregated into different streams, even when stream segregation impedes performance on the task in hand (e.g., Bregman \& Campbell, 1971). Although the strict definition of schemas (Bregman, 1990, p. 398) allows for processes that are learned but automatic, Bregman (1990, p. 400) argues that schemas are intimately connected with attention and should be considered as under attentional control. This definition shall be adopted: Listeners can search for one pattern or another, depending on the task to be done.

A probable role for attention in auditory grouping was noted by Van Noorden (1975). He examined the grouping of a sequence consisting of alternating high- and lowfrequency tones with various tone repetition times (TRTs) and frequency separations. He made a distinction between the fission boundary and the temporal coherence boundary (see Figure 4). Referring to the figure, the sequence could not be heard as a single stream above the temporal coherence boundary. Automatic primitive processes have segregated the sounds, and they cannot be recombined. Below the fission boundary, the sounds could not be separated. Between these two boundaries, either percept could be heard. Primitive stream segregation is influenced by both TRT and frequency separation. At longer TRTs, larger frequency separations are required to induce streaming, as is evident from the slope of the temporal coherence boundary in the figure. In contrast, sounds can be selected by schema-driven processes if their frequency threshold is larger than a minimum critical value, regardless of the TRT. Hence, the fission boundary shown in the figure is almost horizontal.

It is not clear whether the improvement in performance found in Experiment 1 for Condition 2 (timbral contrast), relative to Condition 1 (no difference), was due to enhanced primitive stream segregation or to schema-based 


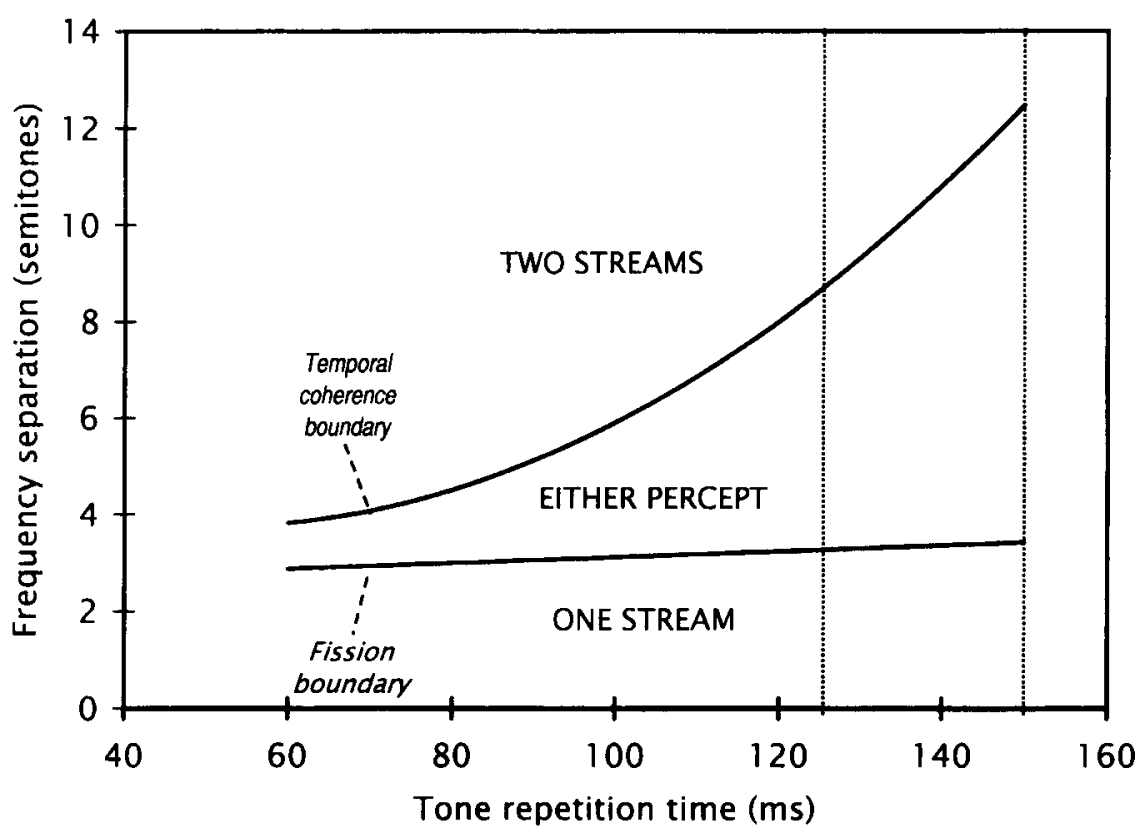

Figure 4. The effect of frequency separation and tone repetition time on the perceptual organization of a sequence comprising higher and lower frequency tones alternating repeatedly (adapted from van Noorden, 1975). The tone repetition time (TRT) is the onset-to-onset interval between successive tones. The vertical dotted lines indicate the TRTs used in our Experiment 1 (left, $125 \mathrm{msec}$ ) and the mean TRT used in our Experiment 2 (right, $150 \mathrm{msec}$ ). From Temporal Coherence in the Perception of Tone Sequences, by L. P. A. S. Van Noorden, 1975. Adapted with permission.

selection. This is because the use of a schema to select sounds with a pure-tone timbre could also lead to the observed difference in performance. To distinguish between these two explanations, a task was designed for Experiment 2 for which good performance depended on the integration of the sequence of sounds (i.e., they had to be heard as a single stream). If the introduction of differences in timbre enhances primitive stream segregation, these differences should lead to worse performance. Moreover, the selection of sounds by top-down schema-driven processes, under attentional control, cannot help to improve performance in this kind of task. Hence, worse performance would indicate the influence of primitive grouping processes.

\section{EXPERIMENT 2}

\section{Method}

Overview. A sequence was played that consisted of high- and low-frequency sounds, alternating repeatedly. In some trials, rhythmic changes were introduced between the high- and the low-frequency sounds, and the listeners were asked to detect these changes. The stimuli were designed so that the rhythmic changes were easy to detect if the high- and low-frequency sounds were heard as a single stream but were difficult to detect if the sounds were allocated to different streams. The effect on performance of a timbral contrast between the high- and the low-frequency sounds was measured.
Subjects. Eight students participated in this experiment of about $45 \mathrm{~min}$. None of the subjects had previously taken part in Experiment 1 . All the subjects reported normal hearing.

Stimuli. Each trial consisted of a single presentation consisting of a sequence of a high- and a low-frequency sound, alternating repeatedly. The structure of two sample trials is shown schematically in Figure 5. In the trial depicted in panel A, the lower and higher frequency sounds are perceived as separate streams, and the slight changes in rhythm are not salient. Panel B shows a trial in which the lower and higher frequency sounds are perceived as a single stream, with their perceptual grouping represented by the diagonal lines. The change in thythm over the course of such trials is salient. All of the sounds were $90 \mathrm{msec}$ in duration, including $10-\mathrm{msec}$ linear onset and offset ramps. The steady-state portions had a level of $72 \mathrm{~dB}$ SPL. Throughout the whole of each trial, the rhythm of the lower frequency sounds remained regular, with their onset-to-onset time fixed at $300 \mathrm{msec}$. For the first eight sounds $(1.2 \mathrm{sec})$ in all of the trials, the higher frequency sounds were always halfway in time between the lower frequency sounds, so the rhythm of the whole sequence was isochronous, with a regular gap of $60 \mathrm{msec}$ after each sound. This period was included to allow time for auditory stream segregation to develop. The extent of streaming for some sequences may continue to increase for more than $30 \mathrm{sec}$, but strong stream segregation can be expected after $1 \mathrm{sec}$ for large frequency separations or faster rates (Anstis \& Saida, 1985). On half of the trials, the isochronous rhythm was continued for the entire trial. On the other half of the trials, over the next 12 sounds, the higher frequency sounds were progressively shifted in time, and the resulting nonisochronous rhythm was then held constant for the remaining 8 sounds. If a higher frequency sound falls at position $n$ in the se- 

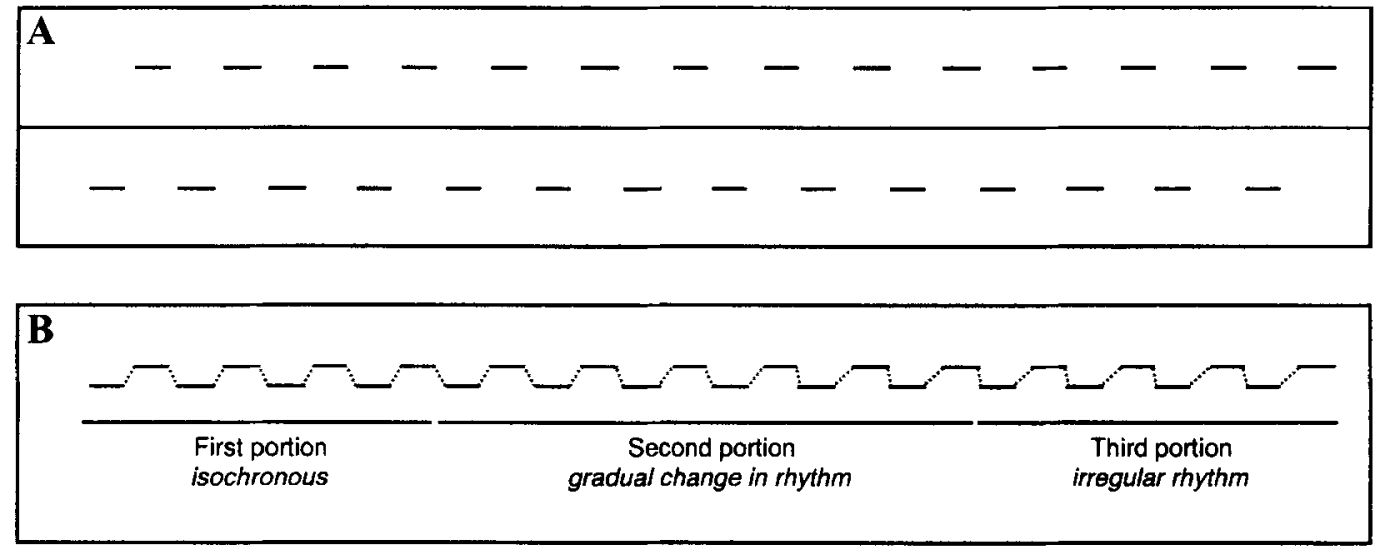

Time

Figure 5. Trial structure of the rhythm task. (A) When the sequence is heard as two streams, the variation in rhythm within each is difficult to detect. (B) When it is heard as a single stream, the altered rhythm toward the end of the sequence is salient. In half of the trials, a change in rhythm was introduced; in the other half, the sequence remained isochronous. The diagonal lines in panel $B$ indicate the perceptual grouping of the sequence when integrated.

quence, its temporal offset, relative to the isochronous rhythm position $(\Delta T)$, can be expressed as follows:

$$
\text { for } \begin{array}{rlrl} 
& n \leq 8 & \Delta T=0 \\
8<n<20 & \Delta T=s(n-8) / 12 \\
n \geq 20 & \Delta T=s,
\end{array}
$$

where

$$
\begin{array}{ll}
s=0 & \text { on regular rhythm trials }(50 \%) \\
s=-36 \mathrm{msec} & \text { on half of the irregular rhythm trials }(25 \%) \\
s=+36 \mathrm{msec} & \text { on the other half }(25 \%) .
\end{array}
$$

The size of the temporal offset was selected on the basis of pilot work, so that the within-stream temporal changes were difficult to detect if the sequence segregated into low- and high-frequency streams but the change in rhythm was salient if the sequence was heard as a single stream. Whether the first sound in the sequence was of higher or lower frequency was randomly determined. The four conditions used are shown in Table 1.

Procedure. The listeners' task was to determine whether the sequence in a trial remained isochronous or became nonisochronous. This was explained with the aid of a diagram. A response was made at the end of each trial. The higher frequency sounds had a center frequency of $800 \mathrm{~Hz}$ in all the trials. The center frequency of the lower frequency sounds was varied adaptively between trials, using a two-up one-down staircase procedure (Levitt, 1971). Larger frequency differences increased stream segregation, making the task more difficult. At the start of each adaptive run, the lower frequency tones were 2 semitones below the higher frequency tones. Throughout the staircase, this frequency separation was constrained to be between 2 and 24 semitones. The initial step size was 1.6 semitones, and this was reduced by a factor of 0.5 after 2 and again after a further 2 turning points. The adaptive run was terminated after 12 turning points, and the mean frequency separation for the last 6 turning points was taken as the threshold.

If timbral contrast induces streaming, it would be expected that a smaller frequency difference would be tolerated for $71 \%$ performance when the timbre of the sounds is different. Hence, it was expected that frequency separation at threshold would be smaller in Conditions 1 and 2 (different timbre) than in Conditions 3 and 4 (same timbre). No difference in performance was expected between Condition 1 and Condition 2 or between Condition 3 and Condition 4.

\section{Results}

The results are shown in Figure 6. To allow for possible key-bias effects, $d^{\prime}$ values were calculated, using the procedure described by Macmillan and Creelman (1991). These were analyzed using a within-subjects ANOVA, which showed that the effect of condition was significant $[F(3,21)=4.2, p<.02]$. Three comparisons were made to test the a priori predictions, using the Bonferroni $t$ test for multiple comparisons. For performance at the threshold level of $71 \%$, a smaller frequency difference was required when the quality of the sounds differed (Conditions 1 and 2, pooled) than when they were the same [Conditions 3 and 4, pooled; $t^{\prime}(15)=2.71, p<.05$ ]. No reliable difference was found between the threshold frequency separations for Conditions 1 and $2\left[t^{\prime}(7)=0.07, p>.50\right]$ or between those in Conditions 3 and $4\left[t^{\prime}(7)=0.67, p>.50\right]$.

\section{Discussion}

The results from this experiment show that differences in timbre can enhance stream segregation even when the listener is trying to integrate the sounds. This implies that a primitive grouping process is the cause of the observed differences in performance. In the conditions used in this experiment, a narrowband noise and a pure tone with a fre-

Table 1

Conditions Used in Experiment 2

\begin{tabular}{cll}
\hline Condition & Lower Frequency Sounds & Higher Frequency Sounds \\
\hline 1 & narrowband noise & pure tone \\
2 & pure tone & narrowband noise \\
3 & narrowband noise & narrowband noise \\
4 & pure tone & pure tone \\
\hline
\end{tabular}




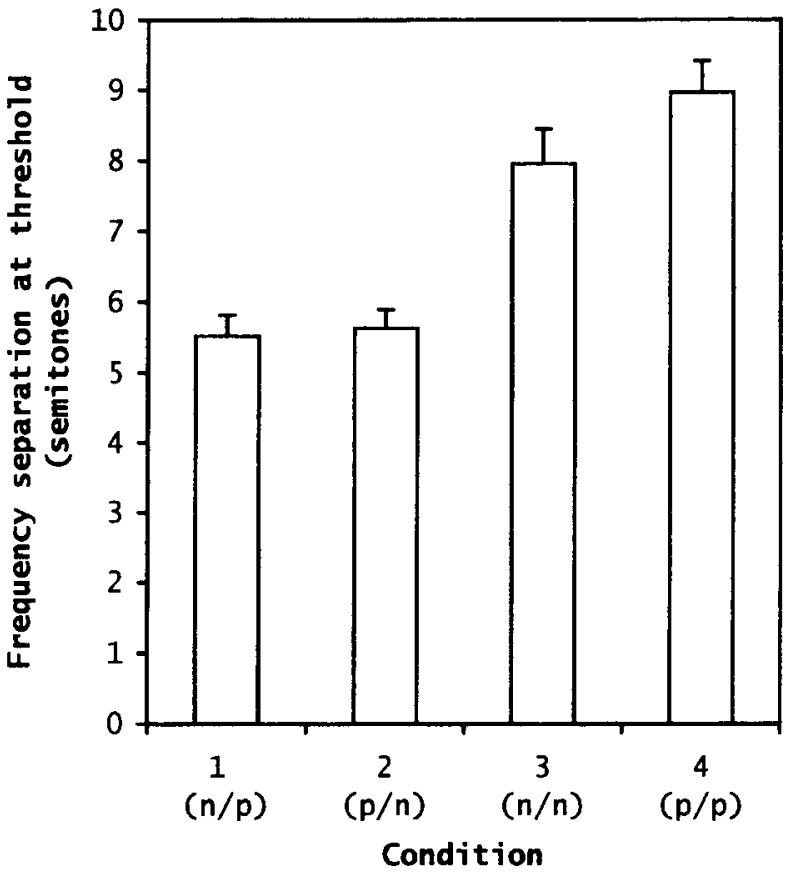

Figure 6. The separation of the lower and higher frequency tones required for threshold $(71 \%)$ performance in the the four conditions of Experiment 2. The letters in parentheses denote the quality ( $n=$ narrowband noise, $p=$ pure tone) of the lower and higher frequency sounds, respectively. The mean performance over 8 subjects, with one standard error, is shown.

quency separation of 5.5 semitones show about the same degree of stream segregation as do two pure tones with a frequency separation of 8.5 semitones. That a reduction in frequency separation of more than a third of the number of semitones was required when the timbres differed suggests that the effects of differences in timbre are appreciable even when compared with the effects of frequency separation. Although the high- and low-frequency sounds used in this experiment fell into different peripheral channels, Hartmann and Johnson's (1991) hypothesis that channeling primarily accounts for stream segregation is not an adequate explanation for these results. This is because differences in timbre were shown to reduce the extent of peripheral channeling required to induce stream segregation.

\section{CONCLUDING DISCUSSION}

The results of the experiments presented confirm, using two performance measures, that differences in the timbres of sounds in a sequence can influence their perceptual organization (see, e.g., Bregman et al., 1990; Iverson, 1995; Singh, 1987; Wessel, 1979). Furthermore, they confirm that stream segregation can occur in the absence of differences in the spectral regions in which energy is present. In Experiment 1, narrowband sounds in the same frequency region were shown to group according to timbre, supporting the conclusions of Dannen- bring and Bregman (1976). Peripheral channeling by frequency (Hartmann \& Johnson, 1991) cannot account for this finding. In Experiment 2, it was again found that there was greater streaming when the sounds had different timbres than when they had the same timbre. Although differences in timbre were accompanied by differences in frequency channeling, those differences were similar across the different conditions. This result parallels those of Singh and Bregman (1997), who also used stimuli that covaried in timbre and frequency channeling. They concluded that manipulations that do not affect peripheral channeling can still affect streaming.

Previous experiments that have explored the effect of differences in timbre on streaming have not attempted to separate the contribution of bottom-up from that of topdown grouping processes. A selective task was used in Experiment 1, and so both bottom-up (primitive stream segregation) and top-down (schema-driven selection) grouping may have been important factors affecting performance. In Experiment 2, it was shown that sounds that differ in timbre may show enhanced streaming even when this streaming impedes the task. This enhancement of streaming must have been a result of bottom-up processes not under attentional control. However, the question of whether or not peripheral channeling is a requirement for primitive stream segregation to operate remains to be answered. Streaming in a condition in which peripheral channeling could not have been a causal factor was only found in Experiment 1. Schema-based selection may have played a role in this experiment; the activity of bottom-up processes was confirmed only in Experiment 2. Hence, it remains possible that selection on the basis of timbre does not require peripheral channeling but that timbral differences can only enhance a primitive stream segregation that has already been induced by peripheral channeling. Although less parsimonious, this explanation merits further research to confirm or refute it.

The implicit assumption here, and in many previous studies, has been that evidence of the facilitation of stream segregation by differences in the acoustic characteristics of sounds should be interpreted as direct effects of timbral similarity. The present study argues against an explanation of these effects simply in terms of peripheral channeling. However, timbre is a multidimensional perceptual property, and it is not necessarily the case that all of its dimensions affect sequential grouping. For example, it may be that some differences between sounds affect their perceived similarity but do not affect the likelihood that sequences of those sounds will stream. To address this issue, research is needed that compares the effects of acoustic differences on tasks designed to measure streaming with the effects of those differences on tasks designed to measure similarity of timbre. Although little research of this kind has been done, a notable exception is the work of Iverson (1995). At least within the range of the stimuli tested, Iverson found evidence to suggest that similarity of timbre was a good predictor of the stream segregation of sound sequences when other factors were controlled. Until more 
studies of this kind have been done, it would seem that the most parsimonious explanation of our findings is that acoustic differences between sounds lead to differences in timbre and that it is these differences that lead to the increased stream segregation observed.

In conclusion, the experiments presented have confirmed that differences in the timbres of sounds can affect their sequential grouping. It has been shown that this effect can occur in the absence of peripheral channeling. Furthermore, it has been shown that primitive stream segregation can be enhanced by differences in timbre.

\section{REFERENCES}

ANSTIS, S., \& SAIDA, S. (1985). Adaptation to auditory streaming of frequency-modulated tones. Journal of Experimental Psychology: Human Perception \& Performance, 11, 257-271.

BeAuvols, M. W., \& MEDDIS, R. (1991). A computer model of auditory stream segregation. Quarterly Journal of Experimental Psychology, 43A, 517-541.

BREgman, A. S. (1978). Auditory streaming is cumulative. Journal of Experimental Psychology, 4, 380-387.

Bregman, A. S. (1990). Auditory scene analysis: The perceptual organization of sound. Cambridge, MA: MIT Press.

Bregman, A. S., \& Campbell, J. (1971). Primary auditory stream segregation and perception of order in rapid sequences of tones. Journal of Experimental Psychology, 89, 244-249.

Bregman, A. S., LiaO, C., \& Levitan, R. (1990). Auditory grouping based on fundamental frequency and formant peak frequency. Canadian Journal of Psychology, 44, 400-413.

Dannenbring, G. L., \& Bregman, A. S. (1976). Stream segregation and the illusion of overlap. Journal of Experimental Psychology: Human Perception \& Performance, 2, 544-555.

Dowling, W. J. (1973). The perception of interleaved melodies. Cognitive Psychology, 5, 322-337.
Glasberg, B. R., \& MOORE, B. C. J. (1990). Derivation of auditory filter shapes from notched-noise data. Hearing Research, 47, 103-138.

HartmanN, W. M., \& JohnSon, D. (1991). Stream segregation and peripheral channeling. Music Perception, 9, 155-183.

IVERSON, P. (1995). Auditory stream segregation by musical timbre: Effects of static and dynamic acoustic attributes. Journal of Experimental Psychology: Human Perception \& Performance, 21, 751-763.

LEVITT, H. (1971). Transformed up-down methods in psychoacoustics. Journal of the Acoustical Society of America, 49, 467-477.

Macmillan, N. A., \& Creelman, C. D. (1991). Detection theory: A user's guide. Cambridge: Cambridge University Press.

MCCABE, S. L., \& Denham, M. J. (1997). A model of auditory streaming. Journal of the Acoustical Society of America, 101, 1611-1621.

Singh, P. G. (1987). Perceptual organization of complex-tone sequences: A tradeoff between pitch and timbre. Journal of the Acoustical Society of America, 82, 886-899.

Singh, P. G., \& Bregman, A. S. (1997). The influence of different timbre attributes on the perceptual segregation of complex-tone sequences. Journal of the Acoustical Society of America, 102, 1943-1952.

VAN NoORdEN, L. P. A. S. (1975). Temporal coherence in the perception of tone sequences. Unpublished doctoral dissertation, Eindhoven University of Technology.

WARrEN, R. M., \& WARrEN, R. P. (1970). Auditory illusions and confusions. Scientific American, 233(6), 30-36.

WESSEL, D. L. (1979). Timbre space as a musical control structure. Computer Music Journal, 3, 45-52.

\section{NOTE}

1. This rate of presentation gives a total duration of $1.25 \mathrm{sec}$ for each interleaved sequence ( 10 notes of $125 \mathrm{msec}$ each without gaps). Although it is true that the tendency to stream may take rather longer than this to build up to a maximum, it should be noted that sequences comprising as few as four tones can segregate perceptually when the tone repetition time is less than $150 \mathrm{msec}$ (Bregman, 1978).

(Manuscript received October 24, 1997; revision accepted for publication May 4, 1999.) 